\title{
Pomerons and Lattices: a Progress Report
}

\author{
UKQCD Collaboration - presented by C. Parrinello ${ }^{\mathrm{a}}$ \\ a Department of Mathematical Sciences, University of Liverpool, \\ Liverpool L69 3BX, United Kingdom
}

We report on some attempts to use lattice QCD to investigate topics in strong interaction phenomenology which are usually interpreted in terms of soft Pomeron exchange.

\section{INTRODUCTION}

It has been known for a long time that the rise of the total hadronic cross section at high energies could be described in the framework of Regge theory via Pomeron exchange [1]. Renewed interest in Pomeron models has been recently triggered by the analysis of diffractive scattering at HERA. In particular, the discovery of events with large rapidity gaps has been interpreted as evidence for Pomeron exchange [2]. Recovering the phenomenology of Pomeron exchange from first principles QCD is a major theoretical problem. Here we describe some attempts to use lattice QCD to test models of diffraction and the Pomeron. We first describe an application of the method to the Landshoff-Nachtmann (LN) model [3] and then we outline a more general approach to the lattice investigation of diffractive physics.

\section{THE LN POMERON}

In the LN model Pomeron exchange between quarks behaves like a $C=+1$ photon-exchange diagram, with amplitude

$i \beta_{0}^{2}\left(\bar{u} \gamma_{\mu} u\right)\left(\bar{u} \gamma^{\mu} u\right)$.

$\beta_{0}$ represents the strength of the Pomeron coupling to quarks, and is related to the (nonperturbative) gluon propagator $D(p)$ by

$\beta_{0}^{2}=\frac{1}{36 \pi^{2}} \int d^{2} p\left[g^{2} D(p)\right]^{2}$,

where $g$ is the gluon-quark coupling. The model yields simple formulae for $p p$ scattering, exclusive $\rho$ production in deep inelastic scattering and the
$J / \Psi$ - nucleon total cross section, which all contain integrals in momentum space of $g^{2} D(p)$ [4].

In order to make predictions one needs an expression for $g^{2} D(p)$. By inserting in the model the gluon propagator as computed on the lattice (in the Landau gauge) we aimed to perform a strong consistency test from the point of view of QCD [5]. For this purpose we generated two sets of quenched configurations and in addition we used data for the gluon propagator as evaluated in [6]. The lattice parameters are listed in Table fif Full details of the results and the method for the determination of the gluon propagator can be found in ref. [5]. In the figures the results obtained from the data sets 1,2 and 3 are plotted with a dotted, solid and dashed line respectively. We inserted in the LN formulae analytical expressions corresponding to the best fits to the lattice gluon propagator on each data set. It turns out that our calculations are not significantly affected by discretisation effects, as all the relevant integrals in momentum space appeared to reach their asymptotic value at an integration scale well below the inverse lattice spacing. It was assumed that in the continuum limit the propagator is multiplicatively renormalisable, as it is in perturbation theory. Also, we neglected the running of the QCD coupling, i.e. we made the approximation $g(p)=g$. As the scale for the momenta in $D(p)$ was set from independent string tension measurements, we only had one free parameter to fix in the expression $g^{2} D(p)$. This is a multiplicative factor corresponding to the product of a gluon wavefunction renormalisation constant times a numerical value for $g^{2}$. We call this parameter $g_{\text {eff }}^{2}$. It was determined by using (2) as 
Table 1

Parameters of the lattices used in the study of the LN model.

\begin{tabular}{lrrrr}
\hline & $\beta$ & Size & Cfgs. & $a^{-1}(\mathrm{GeV})$ \\
\hline Data set 1 & 6.0 & $16^{4}$ & 150 & 1.9 \\
Data set 2 & 6.2 & $16^{4}$ & 150 & 2.7 \\
Data set 3 [6] & 6.0 & $24^{3} \times 48$ & 500 & 1.9 \\
\hline
\end{tabular}

a nonperturbative renormalisation condition, imposing that $\beta_{0}$ attains its phenomenological value of $2.0 \mathrm{GeV}^{-1}$ [7]:

$\beta_{0}^{2}=\frac{1}{36 \pi^{2}} \int d^{2} p\left[g_{\text {eff }}^{2} D_{\text {lat }}(p)\right]^{2}=4 \mathrm{GeV}^{-2}$.

We finally inserted $g_{\text {eff }}^{2} D_{\text {lat }}(p)$ in the formulae of the model. We adopted the analysis procedure of Halzen and collaborators [4].

\subsection{Proton-proton elastic scattering}

The calculation of $\sigma_{\text {tot }}^{0}$ and $\frac{d \sigma^{0}}{d t}$, i.e. the energyindependent part of the total and elastic differential cross section for proton-proton scattering, provides a benchmark for the LN model. Single Pomeron exchange is expected to dominate $\frac{d \sigma}{d t}$ up to $-t \simeq 0.5 \mathrm{GeV}^{2}$. The measured total and elastic differential cross sections are usually parametrised as follows:

$\sigma_{\text {tot }}=\left(\frac{s}{m_{p}^{2}}\right)^{0.08} \sigma_{\text {tot }}^{0}, \frac{d \sigma}{d t}=\left(\frac{s}{m_{p}^{2}}\right)^{0.168} \frac{d \sigma^{0}}{d t}$.

For small $t$, the elastic differential cross section behaves like $e^{B t}$, and the model is characterised by two parameters, $\sigma_{\text {tot }}^{0}$ and $B$.

We computed $\sigma_{\text {tot }}^{0}$ and $B$ on each lattice using the lattice gluon propagator and the effective coupling $g_{\text {eff. }}$. We obtained values of $\sigma_{\text {tot }}^{0}$ ranging from $18.12 \mathrm{mb}$ to $19.85 \mathrm{mb}$ and $B$ from $12.6 \mathrm{GeV}^{-2}$ to $13.6 \mathrm{GeV}^{-2}$, thus in very good agreement with each other, suggesting that both quantities are subject to only small finite volume effects. They are also encouragingly close to the phenomenological values of $\sigma_{\text {tot }}^{0} \simeq 22.7 \mathrm{mb}$ and $B \sim 11 \mathrm{GeV}^{-2}$. In Fig. 1 we show ISR data [8] for the differential elastic cross section at $\sqrt{s}=53 \mathrm{GeV}$ together with the lattice predictions, with the energy correction of Eq. 6 .

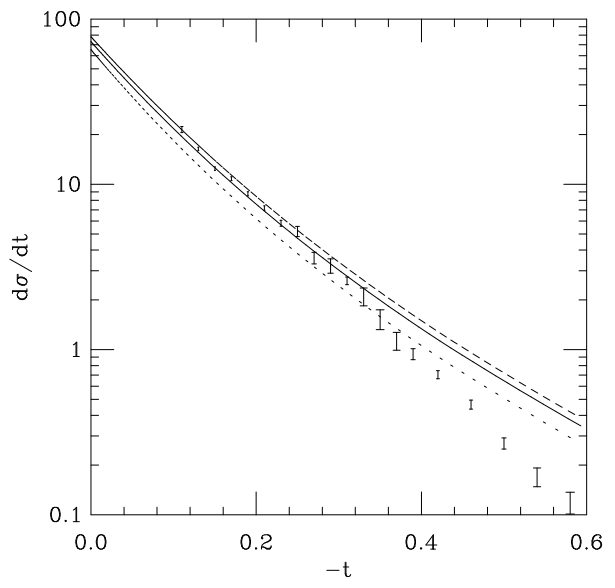

Figure 1. Data for the $p p$ elastic cross section at $\sqrt{s}=53 \mathrm{GeV}$ together with the lattice predictions, corrected for the energy dependence.

\section{2. $J / \psi$-nucleon scattering}

This process provides a further important test of the LN model. Two phenomenological features emerge from our calculations. Firstly, the quarkcounting rule is closely satisfied for light mesons, as we get $\sigma_{p p} / \sigma_{\pi p}$ in the range $1.5-1.8$. Secondly, the Pomeron couples more weakly to heavier mesons, as can be seen from Fig. 2, where we show the meson-nucleon cross section as a function of the pole radius, together with a Regge fit to the energy-independent part of the $\pi^{-} p$ and $K^{-} p$ cross sections 11. Sizeable discrepancies between different lattices can be observed in this case, which may be related to finite volume effects. In this connection, it is worth noting that for most quantities the results obtained from the largest lattice provide the best agreement with 


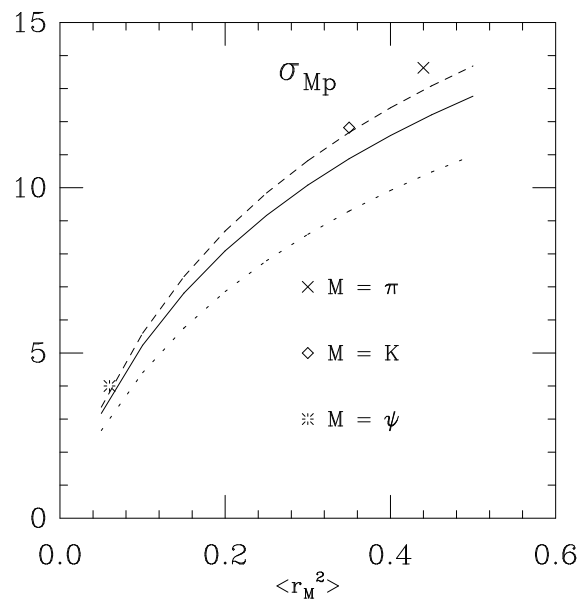

Figure 2. The meson-nucleon total cross section as a function of the radius used in the meson form factor. Also shown are the radii and total cross sections for the $\pi, K$ and $J / \psi$.

the experimental values. In order to further investigate this point we plan to repeat our calculations for a wider range of lattice parameters.

\section{DIFFRACTION ON THE LATTICE}

Here we summarise a more general approach for lattice investigations of diffractive scattering. We start by assuming a factorisable Pomeron exchange. To clarify this concept, consider diffractive scattering at HERA in the one-photon approximation:

$\gamma^{*}(q)+p(p) \rightarrow \tilde{p}(\tilde{p})+X\left(p_{X}\right)$.

If the above process factorises, one can describe it in two steps [9]: first the original proton emits a Pomeron, $p(p) \rightarrow p(\tilde{p})+\mathbb{P}(\Delta)$, then the Pomeron collides with the $\gamma^{*}$ to produce the final hadronic state $X, \gamma^{*}(q)+\mathbb{P}(\Delta) \rightarrow X\left(p_{X}\right)$. On the lattice we aim to study the first subprocess, calculating the effective coupling of the Pomeron to the proton. One motivation for such a study is the investigation of the helicity structure of the Pomeron, as it has recently been argued that a nontrivial spin structure in the Pomeron-proton vertex may affect quantities measured at HERA [9]. Given some ansatz for a composite operator $O_{\mathbb{P}}(x)$ which creates the Pomeron from the QCD vacuum, the effective Pomeron-proton coupling takes the form of a QCD 3-point vertex function, which we can study in momentum space as a function of the momentum of the proton, $p$, and the momentum of the Pomeron, $\Delta$. We assume that $O_{\mathbb{P}}$ only contains gluonic fields. As for the quantum numbers, since the Pomeron is a Regge trajectory all the information we have a priori is that $O_{\mathbb{P}}$ should have a $J^{++}$structure. As we do not want to assign a value to $J$, it makes sense to allow $O_{\mathbb{P}}$ to have arbitrary Lorentz structure, i.e. to be the sum of a scalar, a vector, and tensors of arbitrary rank.

Numerical work is in progress. We are currently focusing on two-gluon operators for $O_{\mathbb{P}}$ with encouraging preliminary results.

\section{ACKNOWLEDGEMENTS}

We acknowledge support from PPARC under grant GR/J21347 and Advanced Fellowships (DGR and CP).

\section{REFERENCES}

1. See for example P.V. Landshoff, hep$\mathrm{ph} / 9605383$ and references therein.

2. See for example M. Derrick et al. (ZEUS coll.), Phys. Lett. B315 (1993) 481.

3. P.V. Landshoff and O. Nachtmann, Z. Phys. C35 (1987) 405.

4. M.B. Gay Ducati et al., Phys. Rev. D48 (1993) 2324 and references therein.

5. D. Henty et al., Phys. Lett. B369 (1996) 130.

6. P. Marenzoni et al., Nucl. Phys. B455 (1995) 339.

7. A. Donnachie and P.V. Landshoff, Nucl. Phys. B244 (1984) 322.

8. A. Breakstone et al., Nucl. Phys. B248 (1984) 253.

9. T. Arens et al., hep-ph/9605376. 